\title{
MODELS OF ROBOT'S WHEEL-MOVER BEHAVIOR ON FERROMAGNETIC SURFACES
}

\author{
Mykyta O. Taranov, Yuriy P. Kondratenko \\ Petro Mohyla Black Sea National University, 10 68th Desantnykiv st., Mykolaiv, Ukraine, \\ mykyta.taranov@chmnu.edu.ua,y_kondrat2002@yahoo.com
}

Paper history:

Received 10 January 2018

Received in revised form 01 March 2018

Accepted 11 March 2018

Available online 31 March 2018

Keywords:

mobile robot;

clamping device;

wheel-mover;

geometrical model;

automatic control system;

simulation.

\begin{abstract}
The mobile robots which can move on complicated working surfaces play a significant role in the automation of various technological processes, in particular, ship repair, fire fighting, inspection of welding quality, rescue operations, etc. This work is a continuation of the authors' investigation of the mobile robot's moving on inclined and vertical ferromagnetic surfaces based on a magnetically operated wheel-mover. Special attention is paid to constructing magnetically operated wheel-mover with twelve legs and modeling of the robot's wheel-mover behavior in different working modes including investigations of the wheel-mover center trajectory, behavior of control signals, etc. Geometrical dependences between a number of wheel-mover legs and deviation of the wheel center path from horizontal line are described. In the present article the modeling results for movement of the wheel-mover on both plain and non-plain surfaces are discussed. For this purpose, the mathematical model of the wheel-mover was created and analyzed and the results were verified using a simulation approach.

Copyright (C) Research Institute for Intelligent Computer Systems, 2018.
\end{abstract}

All rights reserved.

\section{INTRODUCTION}

Robotics, automation and information technologies correlate with production, culture, sport, life science [1] and have very great prospects in different fields of human activity $[1,2]$.

Industrial robots and specialized robotic systems play a significant role in the automation of different industrial technological processes $[2,3]$ in manufacturing, even under various dynamic and uncertain conditions [3]. One of the most important robotics tasks is to control the robot movement [4-6] in an environment with complicated working surfaces [7, 8], with dynamic obstacles and when

(a) the mass of manipulated object is unknown [9],

(b) the robotic system has non-stationary parameters,

(c) the mission of the robotic system can be changed online $[3,10]$.

The tasks and missions of robotic systems define their construction peculiarities and the diversity of their types [2, 7, 8]. Examples are planar robots [11], manipulators with parallel kinematic structure [12], dual arm robot manipulator [13], robots with multiarm structure [14], mobile robots [15-18], etc.
In various industrial branches such as shipbuilding, processing (cleaning, mounting of dowels and explosive devices, firefighting, dyeing, inspection and diagnostics, welding, cutting and polishing and desalination of the ship hulls) of large surfaces or in tight places, human life and health are at risk. The use of mobile robots (MR) increases the productivity as well as has a positive impact on the health of employees.

\section{RELATED WORKS AND PROBLEM STATEMENT}

As the MR can be used in shipbuilding and ship repair, it makes sense to use magnetically operated principles and devices $[19,20]$ in particular, magnetically operated plates and clamping devices. This restricts the range of MR application and requires a powerful clamping force. A magnetically operated wheel-mover (WM) [21] allows the MR to move on surfaces that can be inclined at an arbitrary angle and even have non-planar shape.

The investigations of an interaction between magnetic driver and ferromagnetic surface are discussed in [22]. It is based on the calculation of magnetic field parameters for various thicknesses of 
the layers and creates a mathematical model as well as an algorithm for calculating the mechanical interaction with the permanent magnets.

In [9] authors propose new solutions to creating and improving slip displacement sensors in applications to measure contact forces in robotic systems. In $[23,24]$ a neuro-fuzzy observer of the clamping force was created and the energy efficiency and reliability of the MR moving process was improved.

In [25] the author analyzed current multifunctional MR, such as [26, 27], which can move on inclined and vertical surfaces. Analysis of $[7,8,21,25-27]$ shows that researching of robots with magnetically operated clamping devices is very important and promising for the automation of technological processes in the ship repair industry.

Present researches deal with the development of the structures of MR's intelligent control system [5, 28], the selection of the most acceptable control system components (based on FPGA, for example $[29,30])$, the control algorithms and devices testing in order to model and implement them [31,32] and consideration of the possibility to reconfigure the control system according to a dynamic environment with unknown obstacles or to allow MR's mission to change over time.

Processing of the operations mentioned in the introduction often requires rather high position accuracy of the installed equipment. It is also assumed that most of the operations need to be performed on a curved surface. But there are no research results about meeting these requirements for the WM. This paper aims at considering the mathematical model of the magnetically-operated WM and its behavior on planar and non-planar surfaces and developing approaches for satisfying the equipment requirements.

\section{STRUCTURE OF MAGNETICALLY- OPERATED WHEEL-MOVER}

The structure of the magnetically-operated wheel-mover $[19,20]$ considered in this paper is presented in Fig. 1.

The wheel-mover has $\mathrm{N}$ uniformly allocated spokes 1-4, each with a hinged 5 electromagnet foot 6 that can extend and shorten individually. The length of the legs lies within $\left[R_{\min }, R_{\max }\right]$.

- Rotation is induced by changing the lengths of the spokes.

- Two magnets are attached to a surface 7 permanently.

- It is assumed that the magnets do not slide on the surface.

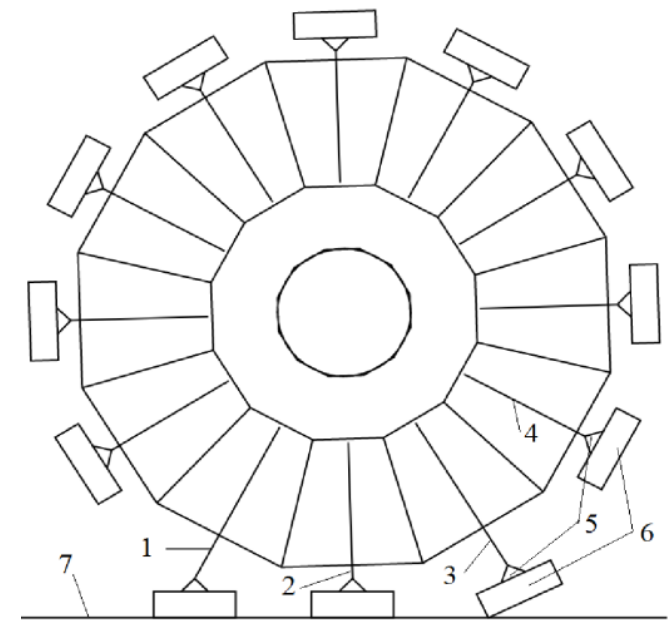

Fig. 1 - The wheel-mover with 12 legs (magnets).

It is supposed that several WMs will be connected to a MR's body similarly to conventional wheels with active suspension.

\section{DESCRIPTION OF A SINGLE STEP OF THE WHEEL-MOVER}

The WM proceeds in individual steps, where each step is made while two spokes are attached to the ground. This section summarizes the results from [29] and continues them by considering features of the WM's movement in the single step case.

\subsection{PATH OF THE WHEEL CENTER}

In [29] the authors obtained conditions on the possible positions of the WM's center $O$ during a single step when two magnets $A, B$ are connected to the surface. The wheel center $O$ lies on the circumcircle of the triangle $A B O$ (Fig. 2) described by formula:

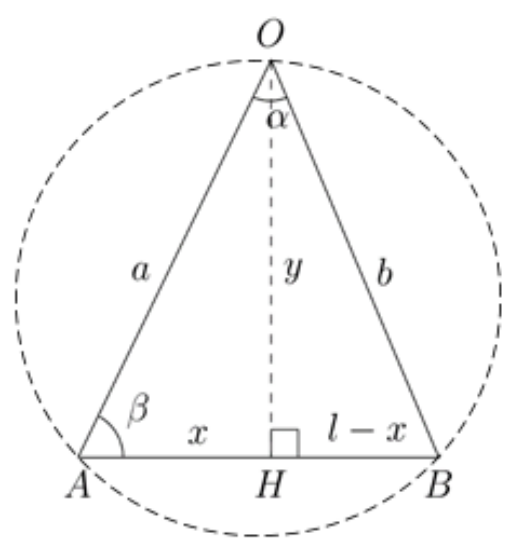

Fig. 2 - Triangle and circumcircle defined by adjacent legs.

$$
\left(x-\frac{l}{2}\right)^{2}+\left(y-\frac{l}{2 \tan (\alpha)}\right)^{2}=\left(\frac{l}{2 \sin (\alpha)}\right)^{2}
$$


As the path of the center of the WM consists of arcs, it cannot move parallel to the surface. Fig. 3 shows a circumcircle of the triangle and the arc $\mathrm{O}_{1} \mathrm{O}_{2}$ of typical positions of $O$ on a plain surface.

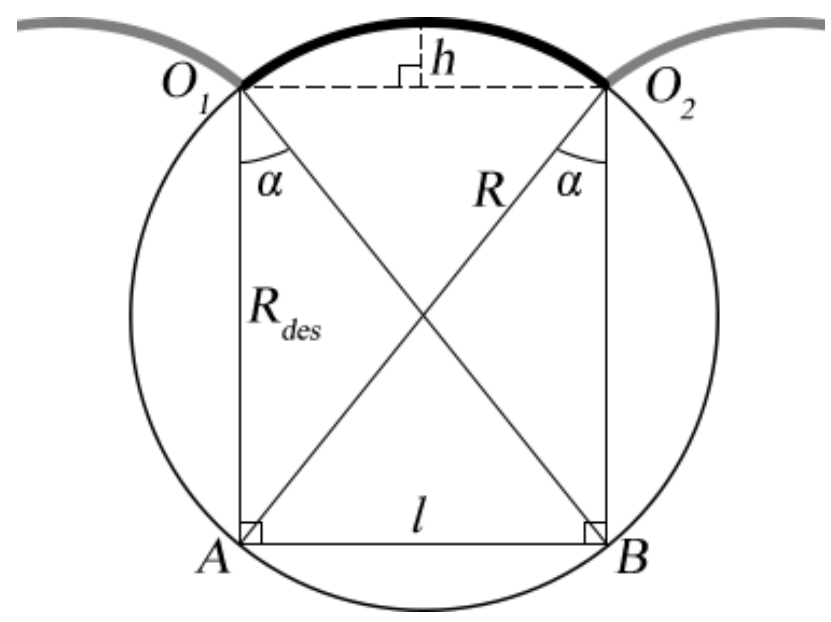

Fig. 3 - Possible positions of the wheel center.

Fig. 4 illustrates the effect of non-constant distance between surface and center of a WM with 12 legs, 8 legs, and 6 legs and $R_{\text {des }}=50$ units.

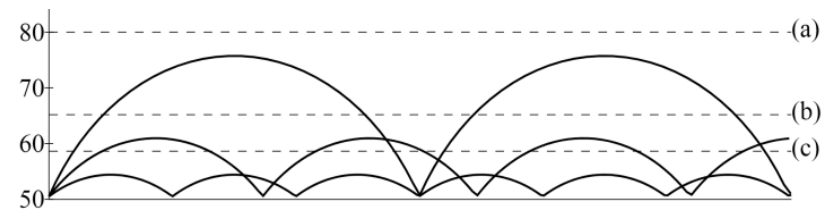

Fig. 4 - Paths in the plane of the wheel center for 6 legs (a), 8 legs (b), and 12 legs (c).

The position of the WM is parametrized in [29] by the angle $\beta$. Of course, there is no direct actor on $\beta$ but by varying the spoke lengths $a$ and $b$, it can be affected. In [29] the lengths of the spokes were calculated as

$$
a=l \frac{\sin (\beta+\alpha)}{\sin (\alpha)} b=l \frac{\sin (\beta)}{\sin (\alpha)} .
$$

\subsection{CHANGES OF Y-COORDINATE OF THE WM CENTER ON A PLAIN SURFACE}

It is assumed that the robot with the WMs will be equipped with the necessary tools. In general, correct processing requires a constant distance between the surface and the equipment. By the construction of the WM this is not possible directly and it is necessary to add mechanical components to compensate for the ripple in the distance. It would be very useful to know the bounds on the ripple at an early design stage.

Figure $\mathrm{ABO}_{2} \mathrm{O}_{1}$ in Fig. 3 is a rectangle, hence $A B=O_{l} O_{2}=l$ holds and the height $h$ of an arc $\mathrm{O}_{1} \mathrm{O}_{2}$ is given by

$$
h=R-\sqrt{R^{2}-\frac{l^{2}}{4}}=\frac{l}{2} \cdot \frac{1-\cos (\alpha)}{\sin (\alpha)}
$$

where $R=l /(2 \sin (\alpha))$ is radius of the circumcircle. By the WM construction $\tan (\alpha)=A B / O_{1} A=l / R_{\text {des }}$, where $R_{\text {des }}$ is a minimal desired leg length during movement on the plain surface. Hence

$$
h=R_{\text {des }} \frac{1-\cos (\alpha)}{\cos (\alpha)}=R_{\text {des }}\left(\frac{1}{\cos \alpha}-1\right) .
$$

Fig. 5 illustrates (4) for fixed $R_{\text {des }}=100$ units and different number of legs $N$. Note that $\alpha=\frac{2 \pi}{N}$. It is seen that the ripple increases in a small number of legs $N$ to rather big values. Thus, for restricting the ripple at design stage one might need to fix a minimal $N$ satisfying this restriction. E.g., twelve legs, which are used in the simulation, provide a change in the $y$-coordinate of $7.74 \%$ of $R_{\text {des }}$. A compensating structure which can extend up to $8 \%$ of $R_{d e s}$ is sufficient to move the robot body parallel to the surface.

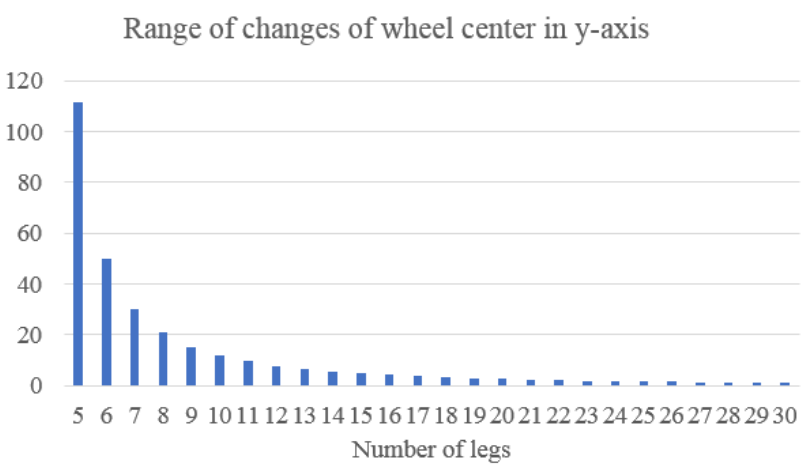

Fig. 5 - Range of changes of $\mathbf{y}$-coordinate for $\boldsymbol{R}_{d e s}=\mathbf{1 0 0}$ units and different number of legs $N$.

\subsection{CONSTANT HORIZONTAL SPEED}

Such processes as welding, cutting and dyeing are very sensitive to speed changes and require a constant speed in a fixed direction. The latter can be provided by special devices compensating unintended movements like the vertical ripple. A constant speed on the other hand can be achieved correspondingly by parameterizing the leg lengths in time.

From Fig. 2 it is obvious that the variables $x$ and $y$ can be expressed in terms of $a$ and $\beta$ by $x=$ $a \cos \beta$ and $y=a \sin \beta$. Horizontal speed is given using addition theorems of trigonometric functions as

$$
\dot{x}^{\prime}=\left(-a \sin \beta+\frac{\partial a}{\partial \beta} \cos \beta\right) \dot{\beta}=\frac{\cos (2 \beta+\alpha)}{\cos \alpha} l \dot{\beta}
$$


with $\frac{\partial a}{\partial \beta}=l \frac{\cos (\beta+\alpha)}{\sin \alpha}$ from (2). This is non-constant for constant angular speed $\dot{\beta}$ which was used in the first implementation of the simulation. Thus, by applying a non-constant angular velocity $\dot{\beta}$ one can obtain a constant horizontal speed $\dot{x}=v$. By integrating (5) from $t_{0}$ to $t$, one obtains

$$
v\left(t-t_{0}\right)=\frac{l\left(\sin (2 \beta(t)+\alpha)-\sin \left(2 \beta\left(t_{0}\right)+\alpha\right)\right)}{2 \cos \alpha} .
$$

This leads to the result

$$
\begin{aligned}
& \beta(t)= \\
& =\frac{\arcsin \frac{l \sin \left(2 \beta\left(t_{0}\right)+\alpha\right)+2 v\left(t-t_{0}\right) \cos \alpha}{l}-\alpha}{2}
\end{aligned}
$$

with $\beta\left(t_{0}\right)$ the angle at the start of the step depending on the configuration of the WM.

\section{THE WM ON NON-PLANAR SURFACES}

Using the WM only on planar surfaces severely limits its scope of operation. Therefore, the features of the WM's movement on non-planar surfaces should also be considered.

To move over longer distances, the WM must make steps to proceed. Each such step is a single step as discussed above. At the end of each step, the WM must have extended one spoke until it touches the surface at some point $C$. To make the step, the rearward spoke must be deactivated and the new-incontact spoke must be activated. It is assumed that this switch happens instantaneously. As a result of the switching the points $A$ and $B$ as well as angle $\beta$ are redefined to the former points $B$ and $C$ and the corresponding angle to reflect the newly activated spokes.

\subsection{MOVING THE WHEEL ON NON-PLANAR SURFACES}

In the planar case the switching of the magnets was timed in such a way that after each switch one spoke was perpendicular to the surface. This can be seen in Fig. 6 , which shows the angle $\beta$ that is within the interval $\left[\frac{\pi}{2}-\alpha ; \frac{\pi}{2}\right]$ for each step.

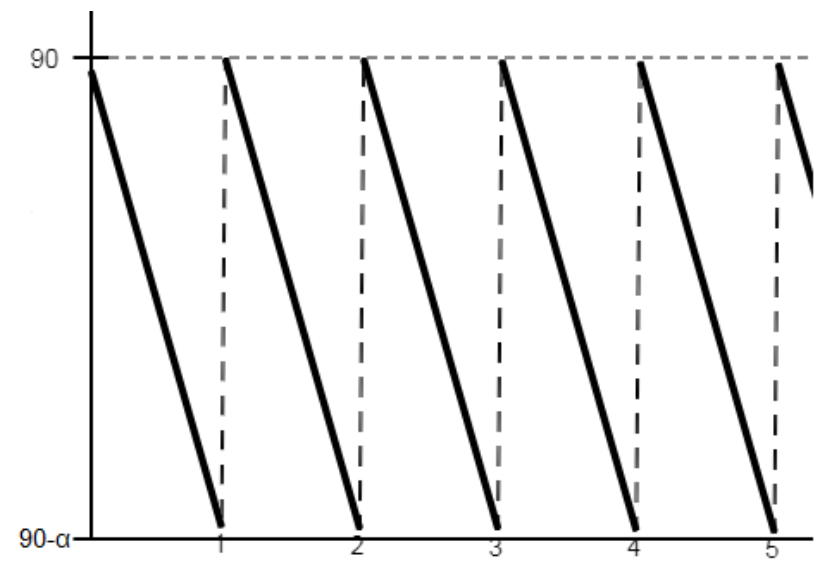

Fig. 6 - Changing the angle $\beta$ on plain surface.

In the non-planar case, the angle $\beta$ does not in general start each step at $\beta=\frac{\pi}{2}$ as sketched in Fig. 6 . Instead the value of the angle $\beta$ and position $x$ at start of each single step must be adopted to the orientation of the WM accordingly.

Moving on a concave surface restricts the interval within which it lies during a single step compared to the planar case. On a convex surface the interval increases. Fig. 7 shows the example result of moving on a non-planar surface.

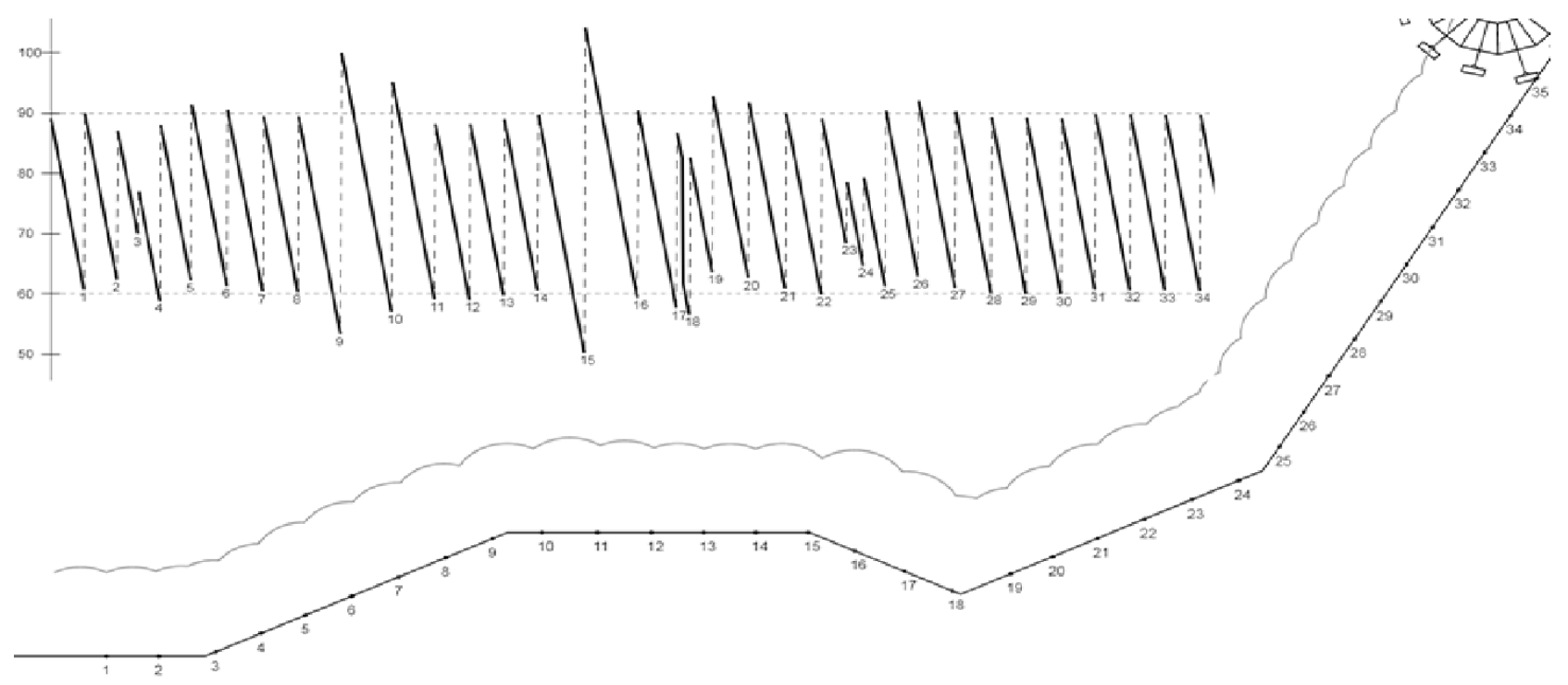

Fig. 7 - Changing the angle $\beta$ on non-plain surface for the wheel with twelve legs. 


\subsection{CRITICAL CURVATURE OF A NON- PLANAR SURFACE}

In this section the authors investigate how much curvature of the surface is allowed, so that the WM can still pass over it.

In [29] it was considered that the WM moves over a convex corner (see Fig. 8) and a critical bend angle described by

$$
\phi_{\text {crit }}=2 \alpha+2 \arcsin \left(\frac{1}{p}\right)
$$

where $p=\frac{R_{\max }}{R_{\min }}$ the ratio of the radii of maximally and minimally extended spokes was calculated.

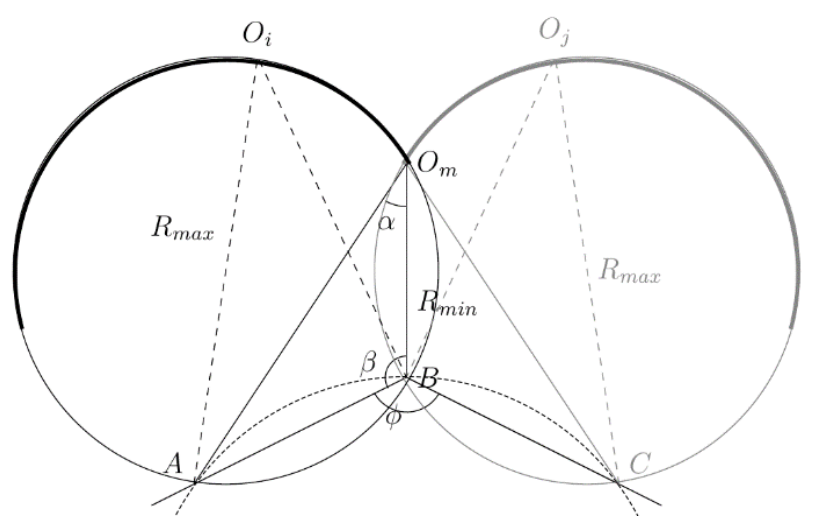

Fig. 8 - Movement of the wheel over the bend.

It is generally accepted to describe a surface geometry by its curvature. The curvature $k=\frac{1}{R}$ is defined as the reciprocal of the radius $R$ of a circle $\Gamma$ that approximates the surface at the particular point.

The curvature of the surface must not exceed a critical curvature $k_{\text {crit }}=\frac{1}{R_{\text {crit }}}$. This is the case if all of the points A, B, C lie on the circle (c.f. Fig. 8). Its radius is then given according to the law of sines by

$$
R=\frac{A B}{2 \sin \left(\frac{\pi-\phi}{2}\right)}=\frac{A B}{2 \cos \left(\alpha+\operatorname{arsin}\left(\frac{1}{p}\right)\right)} .
$$

Applying the law of cosines to the triangle $A B O_{m}$ leads to

$$
A B^{2}=R_{\min }^{2}+p^{2} R_{\min }^{2}-2 p R_{\min }^{2} \cos (\alpha)
$$

Value $\lambda$ is defined as: $\lambda=\frac{R_{\min }}{R}=k R_{\text {min }}$. Setting (10) in (9) leads to

$$
\lambda=\frac{2 \cos \left(\alpha+\arcsin \left(\frac{1}{p}\right)\right)}{\sqrt{1+p^{2}-2 p \cdot \cos (\alpha)}} .
$$

It is favorable to maximize $\lambda$ as this will allow a fixed-sized wheel to pass by a surface of maximal curvature. Note that the problem can be scaled: For a fixed number of legs $N$ with scaled $R_{\min }$ the WM can pass an appropriately scaled circle $\Gamma$.

The function $\lambda(p, N)$ is shown in Fig. 9. According to it, the pretended statement that the larger the leg extension coefficient $p$ is, the more curved the to-be-passed surface can be, is wrong if the legs must be extended completely. For each $N$ there is a critical relative curvature $\lambda_{\text {crit }}(p)$ that represents the maximum relative curvature that the WM can pass. This maximum lies at $p=p_{\text {crit }}(N)$. Values $p>p_{\text {crit }}(N)$ do not allow to pass a higher curvature. E.g. for the WM with $N=20$ legs the maximal $\lambda_{\text {crit }}=1.63$ is located at $p_{\text {crit }}(20)=1.391$. Hence, the legs will extend only up to $39.1 \%$ of $R_{\text {min }}$. Even any larger extension coefficient $p>p_{\text {crit }}(20)$ will not allow the WM to pass by a relative curvature $\lambda>\lambda_{\text {crit }}=1.63$.

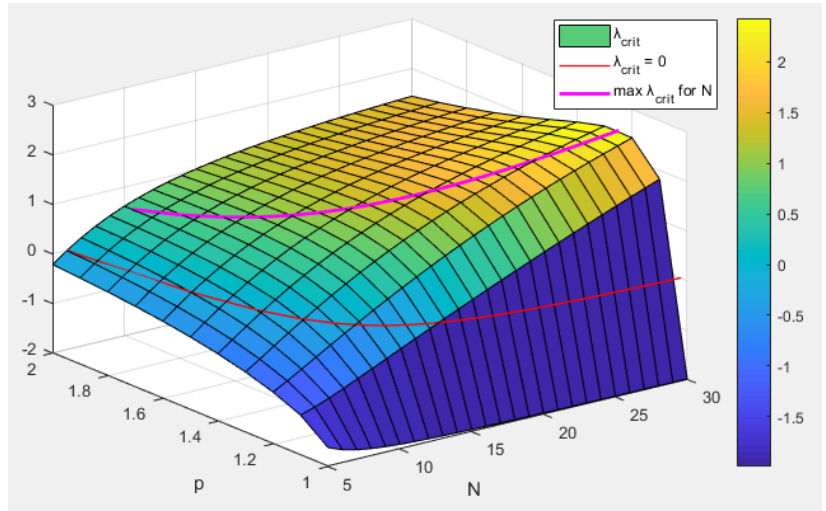

Fig. 9 - The relative curvature $\lambda_{\text {crit }}(p, N)$.

\section{CONCLUSION}

In this work the authors have considered the mathematical model of the magnetically-operated WM and its behavior on planar and non-planar surfaces. These improvements include a sketched scheme to allow the WM to move with a speed whose projection on the planar surface is constant in time. Furthermore, a design tool is given to approximate the possible WM's size considering a given curvature of the surface.

This research was used to improve the simulation of the movement of the WM for different configurations. It can serve as a basis for creating a real automated control system of the wheel-mover and mobile robot.

\section{ACKNOWLEDGMENTS}

The authors gratefully acknowledge the support of this research project by the DAAD (Deutscher Akademischer Austauschdienst), Germany in the 
framework of the Ostpatnershaftprogramme and great thank Prof. Joachim Rudolph and Christian Wolf (Chair of Systems Theory and Control Engineering, Saarland University) for fruitful research cooperation.

\section{REFERENCES}

[1] Y.P. Kondratenko, "Robotics, automation and information systems: future perspectives and correlation with culture, sport and life science, in decision making and knowledge decision support systems," Lecture Notes in Economics and Mathematical Systems, vol.675, A. M. Gil-Lafuente, C. Zopounidis, Eds., Springer, pp. 43-56, 2015. DOI: 10.1007/9783-319-03907-7_6

[2] B. Siciliano, O. Khatib (Eds), Springer Handbook of Robotics, Springer, 2016.

[3] S.B. Niku, Introduction to Robotics: Analysis, Systems, Applications, vol. 7, New Jersey: Prentice Hall, 2001.

[4] Y.-S. Kim, G.-P. Jung, H. Kim, K.-J. Cho, C.-N. Chu, "Wheel transformer: A miniaturized terrain adaptive robot with passively transformed wheels," in Proceedings of the IEEE International Conference on Robotics and Automation (ICRA'2013), Karlsruhe, Germany, May 6-10, 2013, pp. 5605-5610.

[5] M.W. Spong, S. Hutchinson, M. Vidyasagar, Robot Modeling and Control, vol. 3, New York: Wiley, 2006.

[6] C. Collon, J. Rudolph, "Invariant feedback control for the kinematic car on the sphere," Systems \& Control Letters, vol. 61, issue 10, pp. 967-972, 2012.

[7] H. Kim, D. Kim, H. Yang, K. Lee, K. Seo, D. Chang, J. Kim, "Development of a wallclimbing robot using a tracked wheel mechanism," Journal of Mechanical Science and Technology, vol. 22, issue 8, pp. 14901498, 2008.

[8] F. Tache, W. Fischer, R. Moser, F. Mondada, R. Siegwart, "Adapted magnetic wheel unit for compact robots inspecting complex shaped pipe structures," in Proceedings of the IEEE/ASME International Conference on Advanced Intelligent Mechatronics, 4-7 September 2007, pp. 1-6.

[9] Y. Kondratenko, O. Gerasin, A. Topalov, "A simulation model for robot's slip displacement sensors," International Journal of Computing, vol. 15, issue 4, pp. 224-236, 2016, http://www.computingonline.net/computing/art icle/viewFile/854/768.

[10] M. Patil, T. Abukhalil, S. Patel, T. Sobh, "UB Swarm: hardware implementation of heterogeneous swarm robot with fault detection and power management," International Journal of Computing, vol. 15, issue 3, 2016, pp. 162176.

[11] M. Franke, J. Rudolph, \& F. Woittennek, "Motion planning and feedback control of a planar robotic unicycle model," Automation and Robotics, vol. 14, no. 1, pp. 501-506, 2009.

[12] S. Osadchy, V. Zozulya, A. Timoshenko, "The dynamic characteristics of the manipulator with parallel kinematic structure based on experimental data," in Proceedings of the 7th IEEE International Conference on Intelligent Data Acquisition and Advanced Computing Systems: Technology and Applications IDAACS'2015, Berlin, Germany, September 12-14, 2013, vol. 2, pp. 905-911.

[13] C. Park, K. Park, "Design and kinematics analysis of dual arm robot manipulator for precision assembly," in Proceedings of the 6th IEEE International Conference on Industrial Informatics, INDIN 2008, 2008, pp. 430-435.

[14] P. Ji, H. Wu, "Kinematics analysis of an offset 3-UPU translational parallel robotic manipulator," Robotics and Autonomous Systems, vol. 42, issue 2, pp. 117-123, 2003.

[15] G. Campion, G. Bastin, B. Dandrea-Novel, "Structural properties and classification of kinematic and dynamic models of wheeled mobile robots," IEEE Transactions on Robotics and Automation, vol. 12, issue 1, pp.47-62, 1996.

[16] J.C. Alexander, J.H. Maddocks, "On the kinematics of wheeled mobile robots," Autonomous Robot Vehicles, Springer New York, 1990, pp. 5-24.

[17] G. Indiveri, "Swedish wheeled omnidirectional mobile robots: Kinematics analysis and control," IEEE Transactions on Robotics, vol. 25, issue 1, pp. 164-171, 2009.

[18] C. Menon, M. Murphy, M. Sitti, "Gecko inspired surface climbing robots," in Proceedings of the IEEE International Conference on Robotics and Biomimetics, August 22 - 26, 2004, Shenyang, China, 2004, pp. 431-436.

[19] L. Kiltz, C. Join, M. Mboup, J. Rudolph, "Fault-tolerant control based on algebraic derivative estimation applied on a magnetically supported plate," Control Engineering Practice, vol. 26, pp. 107-115, 2014.

[20] M.F. Silva, R.S. Barbosa, A.L.C. Oliveira, "Climbing robot for ferromagnetic surfaces with dynamic adjustment of the adhesion system," Journal of Robotics, vol. 2012, Article ID 906545, 16 pages, 2012, http://dx.doi.org/10.1155/2012/906545. 
[21] M. Taranov, J. Rudolph, C. Wolf, Y. Kondratenko, O. Gerasin, "Advanced approaches to reduce number of actors in a magnetically-operated wheel-mover of a mobile robot," in Proceedings of the XIIIth International Conference on Perspective Technologies and Methods in MEMS Design (MEMSTECH), 2017, pp. 96-100, DOI:10.1109/MEMSTECH.2017.7937542

[22] Y. Kondratenko, Y. Zaporozhets, J. Rudolph, O. Gerasin, A. Topalov, O. Kozlov, "Features of clamping electromagnets using in wheel mobile robots and modeling of their interaction with ferromagnetic plate," in Proceedings of the 9th IEEE International Conference on Intelligent Data Acquisition and Advanced Computing Systems: Technology and Applications IDAACS'2017, Bucharest, Romania, September 21-23, 2017, vol. 1, pp. 453-458.

DOI: 10.1109/IDAACS.2017.8095122.

[23] Y.P. Kondratenko, J. Rudolph, O.V. Kozlov, Y.M. Zaporozhets, O.S. Gerasin, "Neuro-fuzzy observers of clamping force for magnetically operated movers of mobile robots," Technical Electrodynamics, no. 5, pp. 53-61, 2017. (in Ukrainian)

[24] Y.P. Kondratenko, O.V. Kozlov, O.S. Gerasin, Y.M. Zaporozhets, "Synthesis and research of neuro-fuzzy observer of clamping force for mobile robot automatic control system," in Proceedings of the IEEE First International Conference on Data Stream Mining \& Processing (DSMP), 2016, pp. 90-95. DOI: 10.1109/DSMP.2016.7583514.

[25] O.S. Gerasin, "The analysis of features multipurpose mobile robots," Journal of Scientific Papers, Series Computer Technology, Mykolaiv: PMBSSU, vol. 250, issue 238, pp. 25-32, 2014. (in Ukrainian)

[26] D. Longo and G. Muscato, "A small low-cost low-weight inspection robot with passive-type locomotion," Integrated Computer-Aided Engineering, vol. 11, issue 4, pp. 339-348, 2004.

[27] D. Souto, A. Faica, A. Deibe, F. Lopez-Pena, R.J. Duro, "A robot for the unsupervised GritBlasting of ship hulls," International Journal of Advanced Robotic Systems, vol. 9, issue 82, pp. 1-16, 2012.

[28] A. Trunov, "Recurrent approximation as the tool for expansion of functions and models of operation of neural networks," EasternEuropean Journal of Enterprise Technologies, vol. 5/4 (83), pp. 41-48, 2016.
[29] A.V. Palagin, V.M. Opanasenko, S.I. Kryvyi, "Resource and energy optimisation oriented development of FPGA-based adaptive logical networks for classification problem," in V. Kharchenko et al. (Eds), Green IT Engineering: Components, Networks and Systems Implementation. Studies in Systems, Decision and Control, vol. 105, Springer, Cham, pp. 195-218, 2017, DOI: 10.1007/9783-319-55595-9_10

[30] A. Drozd, J. Drozd, S. Antoshchuk, V. Antonyuk, K. Zashcholkin, M. Drozd, O. Titomir, "Green experiments with FPGA," V. Kharchenko et al. (Eds), Green IT Engineering: Components, Networks and Systems Implementation. Studies in Systems, Decision and Control, vol. 105, Springer, Cham, pp. 219-239, 2017, DOI: 10.1007/9783-319-55595-9_12

[31] H. Richter, D. Simon, W. Smith, S. Samorezov, "Dynamic modeling, parameter estimation and control of a leg prosthesis test robot," Appl. Math. Model, vol. 39, pp. 559-573, 2015.

[32] V. Koval, V. Turchenko, A. Sachenko, J.A. Becerra, R.J. Duro, V. Golovko, "Infrared sensor data correction for local area map construction by a mobile robot," The Lecture Notes in Artificial Intelligence, LNAI 2718, Springer-Verlag, Berlin Heidelberg, pp. 306315, 2003.

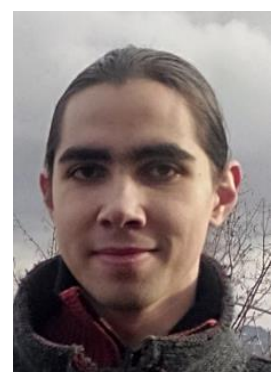

Mykyta Taranov, is a PhD student of Petro Mohyla Black Sea National University (PMBSNU), Ukraine. In 2017 he received the MSc degree in Artificial intelligence systems at PMBSNU.

His research interests include robotics, computer control systems, Internet of things, fuzzy logic.

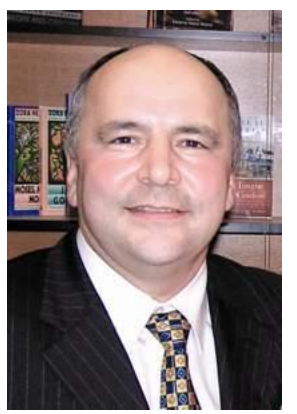

Yuriy Kondratenko DSC, Professor, Fulbright Scholar 2015-16 at Cleveland State University, Honor inventor of Ukraine, professor of the Intelligent Information Systems Department of Petro Mohyla Black Sea National University (PMBSNU).

Scientific interests: robotics, intelligent decision support systems, automatic control, fuzzy sets and fuzzy logic, computer systems and components, modeling and simulation. 